University of Wollongong

Research Online

Faculty of Informatics - Papers (Archive)

Faculty of Engineering and Information

Sciences

$1-1-2002$

\title{
Modelling of color cross-talk in CMOS image sensors
}

Wanqing $\mathrm{Li}$

University of Wollongong, wanqing@uow.edu.au

Philip Ogunbona

University of Wollongong, philipo@uow.edu.au

Yan Shi

University of Wollongong, ys099@uowmail.edu.au

Igor Kharitonenko

University of Wollongong, igor@uow.edu.au

Follow this and additional works at: https://ro.uow.edu.au/infopapers

Part of the Physical Sciences and Mathematics Commons

\section{Recommended Citation}

Li, Wanqing; Ogunbona, Philip; Shi, Yan; and Kharitonenko, Igor: Modelling of color cross-talk in CMOS image sensors 2002.

https://ro.uow.edu.au/infopapers/2138

Research Online is the open access institutional repository for the University of Wollongong. For further information contact the UOW Library: research-pubs@uow.edu.au 


\title{
Modelling of color cross-talk in CMOS image sensors
}

\begin{abstract}
This paper presents a way to model the cross-talk effect in CMOS image sensors. Two algorithms are derived from the model; both of them work on the Bayer raw data and have low computational complexity. Experiments on Macbeth color chart and real images have shown the effectiveness of the modeling to eliminate the cross-talk effect and produce better quality images with traditional color interpolation and correction algorithms designed for CCD image sensors.
\end{abstract}

\section{Keywords}

image, cmos, sensors, talk, modelling, cross, color

Disciplines

Physical Sciences and Mathematics

\section{Publication Details}

Li, W., Ogunbona, P., Shi, Y. \& Kharitonenko, I. (2002). Modelling of color cross-talk in CMOS image sensors. ICASSP, IEEE International Conference on Acoustics, Speech and Signal Processing Proceedings (pp. IV/3576-IV/3579). 


\title{
MODELLING OF COLOR CROSS-TALK IN CMOS IMAGE SENSORS
}

\author{
Wanqing Li, Philip Ogunbona, Yu Shi and Igor Kharitonenko
VIP Lab, Motorola Australian Research Center, Australia
\{wli,pogunbon,yshi,ikhari\}@arc.corp.mot.com

\begin{abstract}
This paper presents a way to model the cross-talk effect in CMOS image sensors. Two algorithms are derived from the model; both of them work on the Bayer raw data and have low computational complexity. Experiments on Macbeth color chart and real images have shown the effectiveness of the modeling to eliminate the cross-talk effect and produce better quality images with traditional color interpolation and correction algorithms designed for CCD image sensors.
\end{abstract}

\section{INTRODUCTION}

Complementary Metal-Oxide-Semiconductor (CMOS) imaging technology $[1,2]$ is emerging as an alternative solid-state imaging technology to charge coupled device (CCD) due to low cost (compatible to standard CMOS technologies), low power consumption and easy integration with other CMOS signal processing modules that would lead to one-chip solution for many applications.

A typical digital color imaging system with one-sensor CMOS imager, as shown in Figure 1, consists of three parts: optical, analogue and digital.

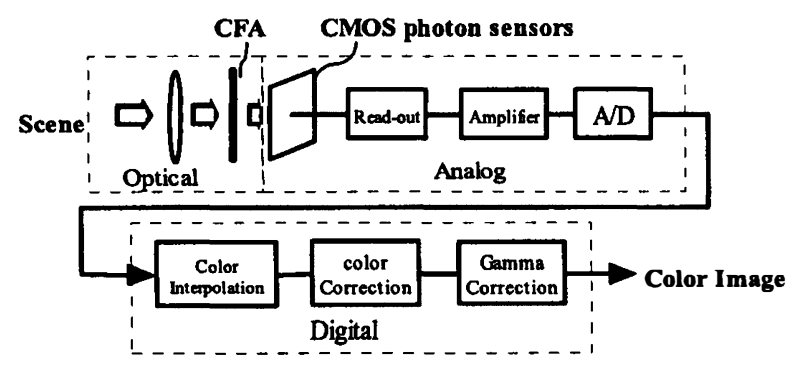

Figure 1. A schematic of one-sensor CMOS imaging system

The analogue part is composed of an array of CMOS sensor elements, read-out circuits, amplifiers and analogue-digital $(A / D)$ converters. The color filter array (CFA) in the optical part is used to filter the incident light such that each sensor element is only exposed to one of the primary colors (Red, Green, and Blue) or one of the complementary colors (Cyan, Yellow and Magenta). Figure 2 gives a typical CFA for RGB primary colors.

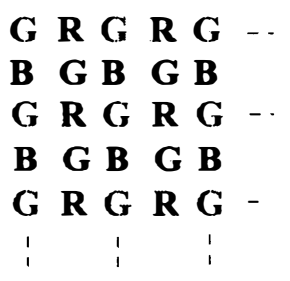

Figure 2. A typical RGB color filter array

Since the primary/complementary colors are only sparsely sampled, i.e. only one of the color components is sampled at each sensor element, recovery of missing colors from the sampled ones is necessary in order to generate a color image. This is usually achieved by color interpolation and correction in the digital processing part.

Compared to CCD image sensors, however, CMOS image sensors often perform less satisfactorily due to its unique problems including dark current, fixed-pattern noise (FPN), pixel cross-talk and high random noise. Though recent improvement in CMOS sensor and circuit technology has combated some of the problems [1] like dark current and FPN, cross-talk [3] and random noise [4] remain unsolved

This paper presents a signal processing based solution to the problem of pixel cross-talk. Section 2 discusses in detail the pixel cross-talk and its impact on a finished color image. In Section 3, a mathematical model and two derived algorithms from the model are described for compensating the pixel cross-talk. Experimental results on both Macbeth color checker and real images are presented in Section 4. The article concludes with some remarks in Section 5.

\section{PIXEL CROSS-TALK}

Pixel cross-talk is a phenomenon wherein neighboring pixels interfere with each other [3]. In other words, the response of the sensor at a given pixel depends not only on the incident light at this pixel, but also on its neighbors. It has been observed that the horizontally adjacent pixels 
interfere with each other much more than vertically adjacent pixels [4] possibly due to the pixel layout.

Considering a CMOS sensor with the RGB CFA as shown in Figure 2, the red pixels interfere with their green neighbors, referred as Gr hereafter, and so do the blue pixels with their green neighbors, referred as $\mathrm{Gb}$ hereafter. As a result of the cross-talk, $\mathrm{Gr}$ and $\mathrm{Gb}$ may appear different even though they receive the same amount of incident light. Figure 3 shows light skin color block from Macbeth color checker and the blocky effect caused by the cross-talk. Its average $\mathrm{Gr}$ and $\mathrm{Gb}$ are 184 and 169 respectively, nearly $10 \%$ difference.

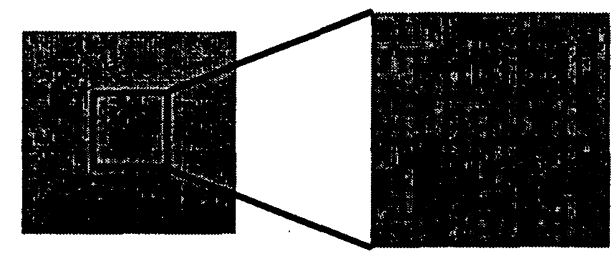

Figure 3. Blocky effect on finished color images caused by cross-talk

There are several possible factors that may contribute to the cross-talk. Optically, light may pass through one pixel filter at such an oblique angle that it strikes its adjacent pixels by the time it propagates down to the sensor surface. Electrically, sensor read-out circuits may allow for the signal read from one pixel to influence the signal read from another pixel. Architecturally, carriers generated by penetrating photons under a pixel diffuse to a nearby pixel depletion region and are collected by the nearby pixel. The depth by which a photon will penetrate a silicon substrate before generating a carrier is strongly wavelength dependent [7] and the longer the wavelength, the deeper the penetration. As a result, the diffusion causes a strong cross-talk between the red pixels and their $\mathrm{Gr}$ neighbors.

To combat the cross-talk problem, a mathematical model is proposed based on the observed characteristics of the cross-talk. The model and two algorithms derived from the model are described in the next section

\section{COMPENSATION OF CROSS-TALK}

From signal processing perspective, cross-talk can be considered as a random noise or noise having certain pattern. Application of median filter or its variations [810] appears to be a straightforward choice for its simplicity. However, median filter is good at removing random impulse noise. The fixed pattern characteristic should be explored as well in order to remove the crosstalk effect effectively.

According to the three hypotheses (physical, electrical and architectural) presented in Section 2 with respect to the source of the cross-talk, the amount of the cross-talk can be estimated locally and its effect can be removed by compensating the difference between the $\mathrm{Gr}$ and $\mathrm{Gb}$ channel.

\subsection{Modeling}

Let us consider a 45-degree diagonal line on which $\mathrm{Gr}$ and $\mathrm{Gb}$ are sampled at every other pixel location. The intensity profiles of these $\mathrm{Gr}$ and $\mathrm{Gb}$ pixels on the line are plotted in Figure 4, where $f_{G r}(x), f_{G b}(x)$ and $f_{G}(x)$ are $\mathrm{Gr}$, $\mathrm{Gb}$ and assumed $\mathrm{G}$ intensity profiles respectively.

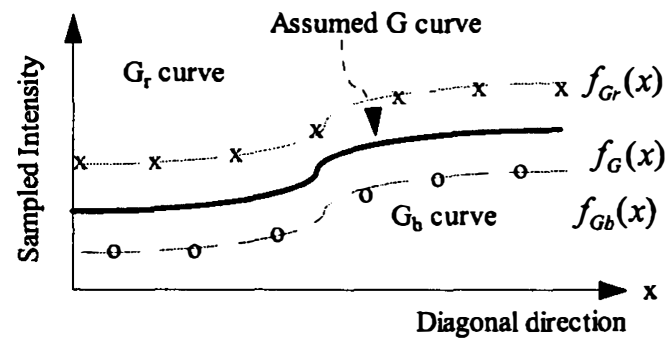

Figure 4 Modeling of cross-talk effect

As a result of the cross-talk, the Gr curve is usually above the $\mathrm{Gb}$ curve. The corss-talk compensation can be formulated as follows

Cross-talk Compensation: To reconstruct $f_{G}(x)$ from $f_{G r}(x)$ and $f_{G b}(x)$, such that the error in gradient between $f_{G}(x)$ and the sampled $f_{G r}(x)$ and $f_{G b}(x)$ is minimized in order to guarantee the sharpness of the image unchanged. That is

$$
f_{G}(x) \propto \min \llbracket 2 \nabla f_{G}(x)-\nabla f_{G r}(x)-\left.\nabla f_{G b}(x)\right|^{2} d x
$$

To solve Equation (1) further assumptions are needed about the $G$ curve. Reasonable assumptions include the local average of the $\mathrm{G}$ curve is close to either the $\mathrm{Gr}$ or $\mathrm{Gb}$ curve or is between the $\mathrm{Gr}$ and $\mathrm{Gb}$ curves.

In the former case, Equation (1) can be solved subject to

$$
\bar{f}_{G}(x)=\bar{f}_{G r}(x)
$$

or

$$
\bar{f}_{G}(x)=\bar{f}_{G b}(x)
$$

where $\bar{f}_{G}(x), \bar{f}_{G r}(x)$, and $\bar{f}_{G b}(x)$ are local averages around $x$.

In the latter case, Equation (1) can be solved subject to

$$
\bar{f}_{G}(x)=\left(\bar{f}_{G r}(x)+\bar{f}_{G b}(x)\right) / 2
$$

the local average can be estimated from the neighborhood of a pixel centered at $x$. 
Without loss of generality, consider the following (shown in Figure 4) $5 \times 5$ local RGB Bayer raw data where $G_{7}$ could either be Gr or Gb. Solutions of Equation (1) can be found using the constrains in Equations (2) or (3).

$$
\begin{array}{llllll}
G_{1} & Y & G_{2} & Y & G_{3} \\
X & G_{4} & X & G_{5} & X \\
G_{6} & Y & G & Y & G_{8} \\
X & G_{9} & X & G_{a} & X \\
G_{b} & Y & G c & Y & G_{d}
\end{array}
$$

Figure 5. A $5 \times 5$ local window from GRBG Bayer pattern.

\subsection{Algorithm I}

Let either constrain (2A) or (2B) be applied. The G channel can be reconstructed by modifying either $\mathrm{Gb}$ or $\mathrm{Gr}$ channel respectively.

With constrain (2B), $G_{r}$ at position $G_{7}$ shall be modified as

$$
G_{7}^{\text {new }}=G_{7}+\Delta G_{7}
$$

where $\Delta G_{7}$ is the average difference of the local average $\mathrm{Gb}$ and its surrounding $\mathrm{Gr}$ pixels.

Notice that only the green values at $G_{r}$ pixels need to be modified using the method described above if constrain (2B) is applied. Similarly, only $G_{b}$ values need to be modified if constrain (2A) is applied.

\subsection{Algorithm II}

Assume condition (3) is applied. The $G$ channel can be reconstructed as follows. For a $\mathrm{G}_{\mathrm{r}}$ pixel

$$
G_{7}^{\text {new }}=G_{7}+\left(\bar{G}_{b}-\bar{G}_{r}\right) / 2
$$

For $\mathrm{a}_{\mathrm{b}}$ pixel,

$$
G_{7}^{\text {new }}=G_{7}+\left(\bar{G}_{r}-\bar{G}_{b}\right) / 2
$$

where $\bar{G}_{r}$ and $\bar{G}_{b}$ are local averages.

\subsection{Color processing chain with $\mathrm{Gr} / \mathrm{Gb}$ compensation}

Since the proposed algorithms work on the Bayer raw data, it must be placed as the first step in the digital color processing chain, as shown in Figure 5. After the crosstalk compensation, most existing color interpolation and correction algorithms can be applied.

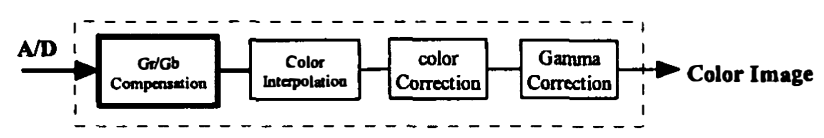

Figure 6 Color processing chain with cross-talk compensation

\section{EXPERIMENTAL RESULTS}

Macbeth color checker and real images captured by a MCM20014 CMOS sensor are used for evaluating the performance of the proposed algorithms. For color interpolation, we applied an edge-based algorithm as described in [6] together with a color correction with $3 \times 3$ matrix.

Table 1 presents the average $\mathrm{Gr}$ and $\mathrm{Gb}$ values of six color boxes from the Macbeth color checker before and after $\mathrm{Gr} / \mathrm{Gb}$ compensation using a media filter and the proposed methods. Notice there is about $10 \%$ difference between $\mathrm{Gr}$ and $\mathrm{Gb}$ channel for the same color. The proposed algorithms removed the $\mathrm{Gr} / \mathrm{Gb}$ difference very well with maximum difference of 1 , which is in some case due to numeric computation error .

The median filter operated on every green pixel and its 4 nearest neighbors. However, it just swapped the $\mathrm{Gr}$ and $\mathrm{Gb}$ channel (column M-flt). This is because at every $\mathrm{Gr}$ pixels, there are 4 nearest $\mathrm{Gb}$ pixels and every $\mathrm{Gb}$ pixel has 4 nearest $\mathrm{Gr}$ pixels.

Figure 7, 8, 9 are the finished Macbeth color checker without $\mathrm{Gr} / \mathrm{Gb}$ compensation and with compensation using Algorithm I and algorithm II respectively. The blocky effect in Figure 7 is usually not noticeable until it's zoomed in. Therefore, a small block of the yellow color was zoomed in by a factor of 3 .

Figure 10,11 and 12 are real images without $\mathrm{Gr} / \mathrm{Gb}$ compensation and with $\mathrm{Gr} / \mathrm{Gb}$ compensation using the proposed algorithm I and II respectively.

Table 1 The average $G r$ and $G b$ values of 5 color boxes from Match color checker before and after $\mathbf{G r} / \mathbf{G b}$ compensation

\begin{tabular}{|l|l|c|c|c|c|}
\hline \multirow{2}{*}{ Color } & \multirow{2}{*}{ Before } & \multicolumn{3}{c|}{ After } \\
\cline { 4 - 6 } & & M-flt & Alg I & Alg II \\
\hline Skin & Gr & 184 & 169 & 170 & 177 \\
\cline { 2 - 6 } & Gb & 169 & 184 & 169 & 176 \\
\hline Blue & Gr & 18 & 20 & 20 & 19 \\
\cline { 2 - 6 } & Gb & 20 & 18 & 20 & 19 \\
\hline Green & Gr & 95 & 92 & 92 & 93 \\
\cline { 2 - 6 } & Gb & 92 & 95 & 92 & 93 \\
\hline \multirow{2}{*}{ Red } & Gr & 71 & 57 & 58 & 64 \\
\cline { 2 - 6 } & Gb & 57 & 71 & 57 & 64 \\
\hline \multirow{2}{*}{ Grey } & Gr & 207 & 199 & 199 & 203 \\
\cline { 2 - 6 } & Gb & 199 & 206 & 199 & 203 \\
\hline
\end{tabular}




\section{SUMMARY}

We proposed two simple and efficient algorithms for removing the cross-talk effect in CMOS image sensors without degrading the sharpness of the images. The algorithms work only on the Green channel of the Bayer raw data.

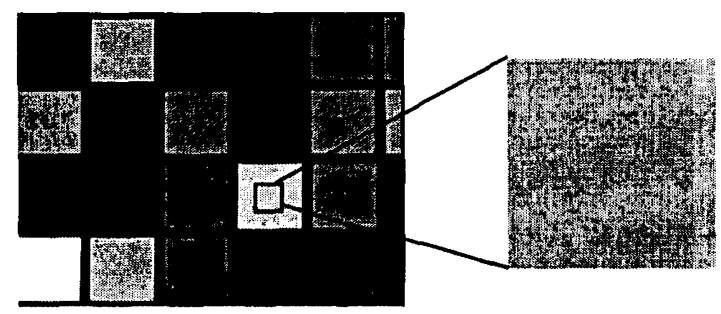

Figure 7 Without $\mathbf{G r} / \mathbf{G b}$ compensation

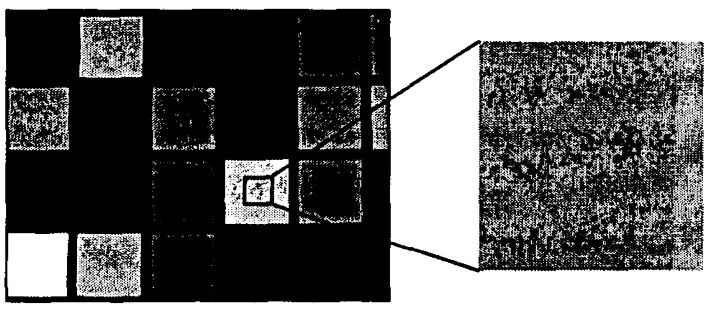

Figure 8 With $\mathbf{G r} / \mathbf{G b}$ compensation using Algorithm I

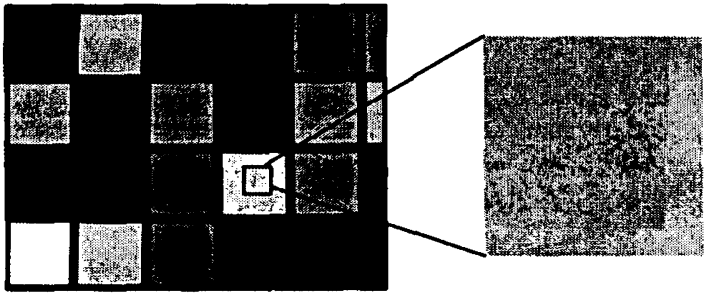

Figure 9 With Gr/Gb compensation using Algorithm II

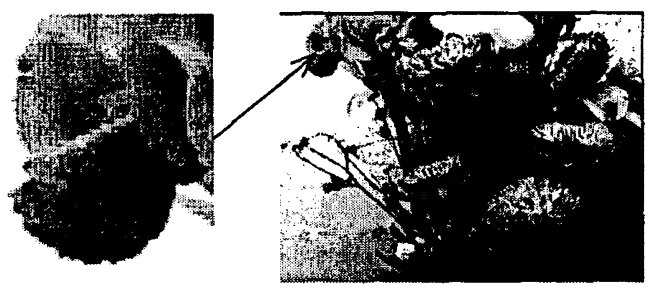

Figure 10 Without $\mathbf{G r} / \mathbf{G b}$ compensation

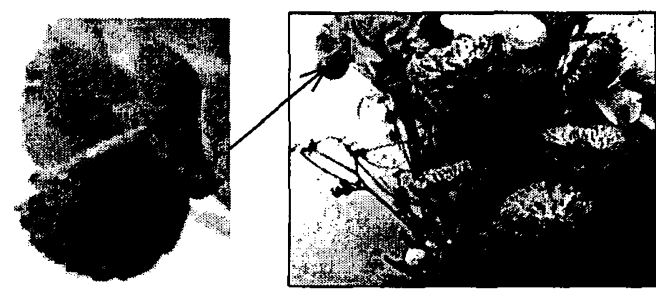

Figure 11 With $\mathbf{G r} / \mathbf{G b}$ compensation using Algorithm I

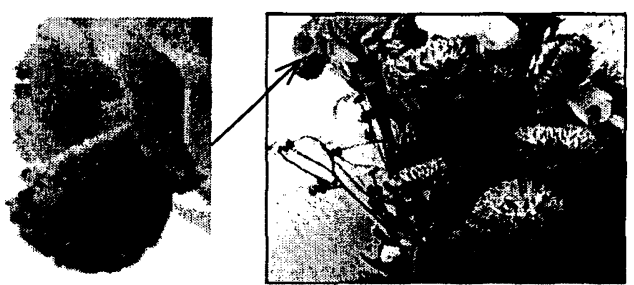

Figure 12 With Gr/Gb compensation using Algorithm II

\section{REFERENCES}

[1] M. J. Loinaz, K. J. Singh, A. J. Blanksby, D. A. Inglis, K. Azadet, and B. D. Ackland, "A 200-mV, 3.3-V, CMOS color camera IC producing $253 \times 28824-\mathrm{b}$ video at 30 framees/s", IEEE Journal of Slid-State Circuits, 33(12), pp.2092-2103, 1998.

[2] H. S. Wong, "Technology and device scaling considerations for CMOS imagers", IEEE Trans. Electron Devices, 43(12), pp.2131-2142, 1996.

[3] A. J. Blanksby and M. J. Loinaz, "Performance analysis of a color CMOS photogate image sensor", IEEE Trans Electron Devices, 47(1), pp.55-64, 2000.

[4] H. Tian, B. Fowler and A. E. Gamal, "Analysis of temporal noise in CMOS photodiode active pixel sensor", IEEE Journal of Solid-State Circuits, 36(1), pp.92-101, 2001.

[5] J. Adams, K. Parulski and K. Spaulding, "Color processing in digital cameras", IEEE MICRO, pp.29, November-December 1998

[6] J. E. Adams, "Interactions between color plane interpolation and other image processing functions in electronic photography", Proc SPIE, vol.2416, SPIE-Int'1 Sco. For Optical Engineering, Bellingham, Wash., pp.144-155, 1995.

[7] J P. Lavine, E. A. Trabka, B. C. Burkey, T. J. Tredwell, E. T. Nelson and C. Anagnostopoulos, "Steady-state photocarrier collection in silicon imaging devices", IEEE Trans Electron Devices, ED-30(9), pp.1123-1134, 1983.

[8] R. T. Chin and C. L. Yeh, "Quantitative evaluation of some edge-preserving noise smoothing techniques", Computer Vision, Graphics and Image Processing, vol.23, pp.67-91, 1983.

[9] X. Wanq, "Adaptive multistage median filter", IEEE Trans. Signal Processing, 40(4), pp.1015-1017, 1992.

[10] A. Beghdadi and A. Khelhaf, " A noise-filtering method using a local information measure", IEEE Trans. Image Processing, 6(6), pp.879-882, 1997. 